\title{
EFECTO DEL EJERCICIO MODERADO Y CONTINUO FRENTE AL ESTRÉS OXIDATIVO INDUCIDO EN Rattus norvegicus WISTAR
}

\author{
Effect of the moderate and continuous exercise induced in Rattus norvergicus Wistar
}

\author{
Elena R. Benavides, Carlos E. Cabrera, Obed J. Ahumada, Elsa E. Robles
}

Departamento de Bioquímica, Facultad de Farmacia y Bioquímica. Universidad Nacional Mayor de San Marcos. Lima - Perú

\section{RESUMEN}

El presente trabajo se realizó con el objetivo de demostrar experimentalmente que el ejercicio moderado y continuo tiene efecto antioxidante, contrarrestando el estrés oxidativo inducido. Con este fin se utilizó un diseño experimental transversal de tipo casos y controles utilizando 32 ratas Wistar de 25 g de peso cada una y un mes de vida, distribuidas en 4 grupos: "A" Control, "B" inducido a estrés, "C" inducido a estrés y sometido a ejercicio físico moderado y continuo, "D" sometido sólo a ejercicio físico moderado y continuo. El estrés fue provocado por la inhalación de etanol y posterior administración de anfetamina (o,5 mg/Kg de peso) durante siete días. Se sometió a las ratas a ejercicio moderado y continuo por siete días. Se sacrificaron los animales y se evaluó la actividad antioxidante de las enzimas Superóxido Dismutasa (SOD) y Catalasa (CAT) en hígado y cerebro, obteniéndose los siguientes resultados: grupo A, hígado: 32,89 x 10 $0^{-3}$ UCAT/mg proteína y 9,99 x 10 ${ }^{-3}$ USOD/mg proteína; cerebro: 5,99 UCAT/mg proteína y o,37 USOD/mg proteína; grupo B, hígado: $27 \times 10^{-3} \mathrm{UCAT} / \mathrm{mg}$ proteína y $7,63 \times 10^{-3} \mathrm{USOD} / \mathrm{mg} \mathrm{proteína;} \mathrm{ce-}$ rebro: 4,64 UCAT/mg proteína y o,26 USOD/mg proteína; grupo C, hígado: 40,54 X 10 ${ }^{-3} \mathrm{UCAT} / \mathrm{mg}$ proteína y 9,21 x 10 ${ }^{-3}$ USOD/ mg proteína; cerebro: 7,97 UCAT/mg proteína y o,37 USOD/mg proteína. Se concluye que en las condiciones experimentales el Grupo B sufrió estrés oxidativo como producto del tratamiento y el Grupo C se recuperó gracias al ejercicio moderado.

Palabras clave: Estrés oxidativo, anfetaminas, antioxidantes, ejercicio moderado y continuo.

\section{SUMMARY}

The objective of the present research is demostrate in an experimental way that the continuos moderate exercise has an antioxidant effect reducing the provoked stress. With this clear objective we designed a transversal experiment using case studies with a controlled group using 32 Wistar rats with 25 grams each one and with one month old too. All these rats were distributed in four groups: "A" was the control one, "B" was induced to have stress, "C" was induced to have stress with continuos moderate physical exercise, "D" was under continuos moderated physical exercise. The stress was provoked by ethanol inhalation and some doses of amphetamine (o,5mg/Kg of weight) for 7 days. The rats were under continuos moderated exercises for 7 days. The animals were sacrificed and the antioxidant activity of Superoxido Dismutase (SOD) and Catalasa (CAT) in liver and brain was tested, obtaining the following results: group A, liver: $32,89 \times 10^{-3} \mathrm{UCAT} / \mathrm{mg}$ protein \& 9.99 x 10 ${ }^{-3} \mathrm{USOD} / \mathrm{mg} \mathrm{protein}$; brain: 5,99

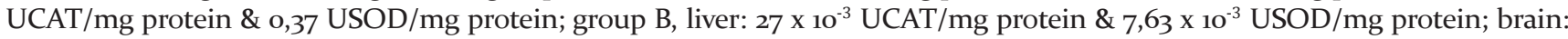
4,64 UCAT/mg protein \& o,26 USOD/mg protein; group C, liver: 40,54 X 10 $0^{-3} \mathrm{UCAT} / \mathrm{mg}$ protein \& 9,21 x 10 ${ }^{-3} \mathrm{USOD} / \mathrm{mg}_{\mathrm{protein}}$; brain: 7,97 U CAT/mg protein \& o,37 U SOD/mg protein. Therefore, we conclude that these experimental conditions Group B suffered oxidative stress as a result of treatment, and the Group $C$ achieved a recovery thanks to continuos moderate exercise.

Key words: Oxidative stress, amphetamines, antioxidants, moderate exercise and continuous.

\section{INTRODUCCIÓN}

L os radicales libres $(\mathrm{RL})$ son entidades químicas con uno o más electrones libres en su última órbita, lo que los hace inestables y altamente reactivos, le sustraen un electrón a la molécula vecina oxidándola y transformándola en un nuevo RL. Todos los seres vivos que utilizan el oxígeno para generar energía liberan RL y en las células existen sistemas de defensa que neutralizan a estas moléculas, como las enzimas antioxidantes Catalasa (CAT), Superóxido Dismutasa (SOD) y Glutatión Peroxidasa (GPx).

Los RL y los antioxidantes corporales se encuen- tran en equilibrio; cuando estos radicales libres se acumulan y superan la barrera antioxidante se llega al estrés oxidativo (EO), el cual está asociado a la aceleración del envejecimiento y diversas patologías degenerativas.

El ejercicio posee un rol controversial en la producción de RL. El ejercicio físico agudo, es decir extenuante, está asociado con el incremento en la generación de radicales libres, principalmente debido al dramático incremento en el consumo de oxígeno ${ }^{(1)}$. El ejercicio extenuante causa estrés oxidativo en personas sedentarias. Un ejercicio "de fin de semana” podría entrar en contradicción con las creencias populares, siendo no beneficioso. Se señala además, que un ejercicio regular podría prevenir diversos males por decreci- 
miento de la peroxidación lipídica, decrecimiento del daño muscular y elevación del sistema antioxidante ${ }^{(2,3,4)}$.

Existe evidencia de que el ejercicio físico regular y no agotador tiene efectos benéficos, pero estos desaparecen con el agotamiento y la falta de entrenamiento porque durante el ejercicio físico agotador se genera tal cantidad de radicales libres que las defensas antioxidantes se ven incapaces de prevenir el daño que éstas inducen ${ }^{(5)}$.

Sin embargo, si el ejercicio es controlado y se acompaña con la respiración adecuada se experimenta una sensación de bienestar, por lo que se planteó el objetivo de evaluar experimentalmente el efecto del ejercicio moderado en la producción de RL a través de la medición de algunos de los indicadores en un modelo animal de tipo transversal.

\section{MATERIALES Y MÉTODOS}

El material biológico estuvo constituido por 32 ratas Wistar con un peso aproximado de $25 \mathrm{~g}$ cada una, de un mes de vida, adquiridas en el Centro de Control de Chorrillos del Instituto Nacional de Salud y mantenidas con alimentación y bebida ad-libitum en el bioterio de la Facultad de Farmacia y Bioquímica de la UNMSM durante siete días para su aclimatación; distribuidas posteriormente en cuatro grupos: A, o Control, no sometidas a tratamiento alguno; B, inducidas a estrés oxidativo; C, inducidas a estrés oxidativo y luego a ejercicio moderadoy continuo; D, sometidas a ejercicio moderado y continuo.

El trabajo se desarrolló en tres etapas: (1) inducción a estrés oxidativo, (2) tratamiento antioxidante mediante el ejercicio moderado y continuo, y (3) determinación de la actividad de las enzimas antioxidantes CAT y SOD, tanto en hígado como en cerebro, órganos claves involucrados en el metabolismo y en el ejercicio físico.

\section{Inducción a estrés oxidativo}

La inducción a EO se realizó por exposición a vapores de etanol, 3 horas diarias por 7 días para cada una de las ratas de los grupos B y C alojadas en cámaras de saturación en grupos de 8 . Transcurridas 24 horas desde la exposición a etanol, se les administró anfetamina por vía intraperitoneal a una dosis de $0,5 \mathrm{mg} / \mathrm{Kg}$ de peso.

\section{Tratamiento antioxidante mediante ejerci- cio moderado y continuo}

El ejercicio al que fueron sometidas las ratas se hizo en un equipo que consta de un soporte de acero con ruedas de $19 \mathrm{~cm}$ de diámetro x $6 \mathrm{~cm}$ de ancho, movidas por un motor eléctrico.
Metodología. El ejercicio físico continuo y moderado se consiguió colocando a cada rata en la rueda, la cual se hacía girar por 30 minutos a una velocidad de 60 rpm dos veces al día, durante 7 días, propiciando que el animal trote.

\section{Determinación de la actividad de las enzi- mas antioxidantes}

\section{Procesamiento de las muestras}

Se prepararon homogenizados de hígado y de cerebro de cada animal, utilizando por cada órgano completo $5 \mathrm{~mL}$ de buffer fosfato $0,5 \mathrm{M} \mathrm{pH} 7$; determinándose en cada uno de éstos la concentración de proteínas por el método de Biuret para referir los resultados de actividad en unidades por mg de proteína ${ }^{(6-9)}$.

\section{Determinación de Superóxido Dismutasa}

Fundamento. La reacción se basa en la capacidad de la SOD de inhibir la autooxidación de la epinefrina al compuesto coloreado adrenocromo.

Metodología. Se diluyeron o, $5 \mathrm{~mL}$ de homogeneizado en csp $2 \mathrm{~mL}$ de agua destilada y a partir de esta dilución se midieron $40 \mu \mathrm{L}$ para $1 \mathrm{~mL}$ (dilución final), luego se procedió como sigue:

\begin{tabular}{|lcc|}
\hline \multicolumn{1}{|c}{ Reactivos } & Problema & Blanco \\
Buffer carbonato/EDTA 0,1M pH 10,2 & $0,9 \mathrm{~mL}$ & $0,98 \mathrm{~mL}$ \\
Muestra (Dilución final) & $0,08 \mathrm{~mL}$ & - \\
Epinefrina & $0,01 \mathrm{~mL}$ & $0,01 \mathrm{~mL}$ \\
\hline
\end{tabular}

Las lecturas se hicieron a 480 ๆm; leyéndose las absorbancias $\mathrm{A}_{1}$ a los 30 segundos y $\mathrm{A}_{2}$ a los 210 segundos ${ }^{(8)}$.

\section{Determinación de Catalasa}

Fundamento. La descomposición del $\mathrm{H}_{2} \mathrm{O}_{2}$ puede ser seguida directamente por la disminución de la absorbancia a $240 \mathrm{\eta m}$. La diferencia en absorbancia por unidad de tiempo es una unidad de medida de catalasa.

Metodología. Se diluyó la muestra 1:1 con buffer fosfato $\mathrm{pH}$ 7. Se leyeron las absorbancias $\mathrm{A}_{1}$ y $\mathrm{A}_{2}$ a los 30 y 150 segundos, respectivamente, luego del siguiente tratamiento ${ }^{(8)}$ :

\begin{tabular}{|ccc|}
\hline Reactivos & Muestra & Blanco \\
Sol. $\mathrm{H}_{2} \mathrm{O}_{2} 30 \mathrm{mM}$ & $1,0 \mathrm{~mL}$ & $1,0 \mathrm{~mL}$ \\
Incubar a $25^{\circ} \mathrm{C}$ por 5 minutos & \\
Muestra diluida & $0,3 \mathrm{~mL}$ & - \\
\hline
\end{tabular}

\section{RESULTADOS}

Los valores promedio de SOD, en hígado y cerebro, de cada grupo evaluado se grafican en las figuras 1 


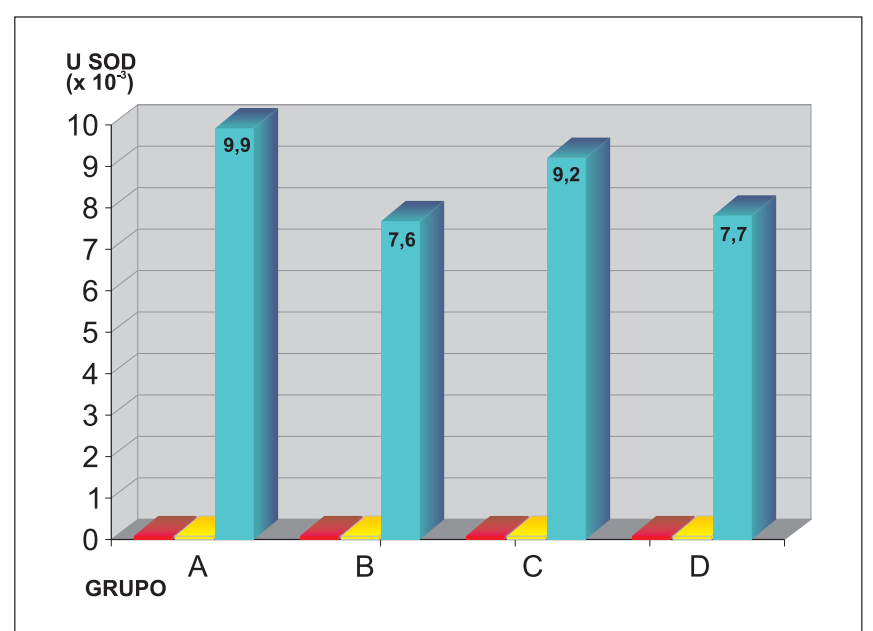

Figura 1. Superóxido Dismutasa en Hígado

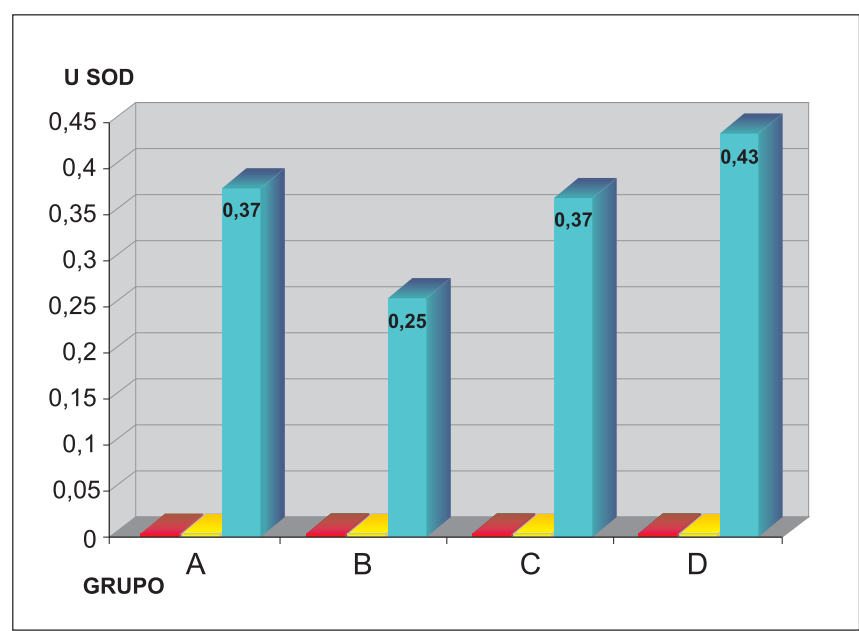

Figura 2. Superóxido Dismutasa en Cerebro

y 2 , respectivamente, mientras que los correspondientes a CAT, en el mismo orden, se muestran en las figuras 3 y 4 .

\section{DISCUSIÓN}

La actividad enzimática de SOD y CAT en el grupo A es la normal, ya que dicho grupo no fue sometido a tratamiento alguno; se considera con estrés oxidativo, a los animales de experimentación del grupo $\mathrm{B}$ que presentaron una actividad de SOD y CAT menor que el promedio del grupo $\mathrm{A}$, mientras que los del grupo $\mathrm{C}$ mostraron mejores resultados evidenciando una notable recuperación luego de la evaluación estadística, para un $\alpha=0.05$ se encontró diferencia significativa entre los grupos $C$ y D. La determinación de la actividad de las enzimas antioxidantes, expresadas por miligramos de proteínas, elimina las diferencias en peso y estado nutricional que pudieran presentar los animales de experimentación.

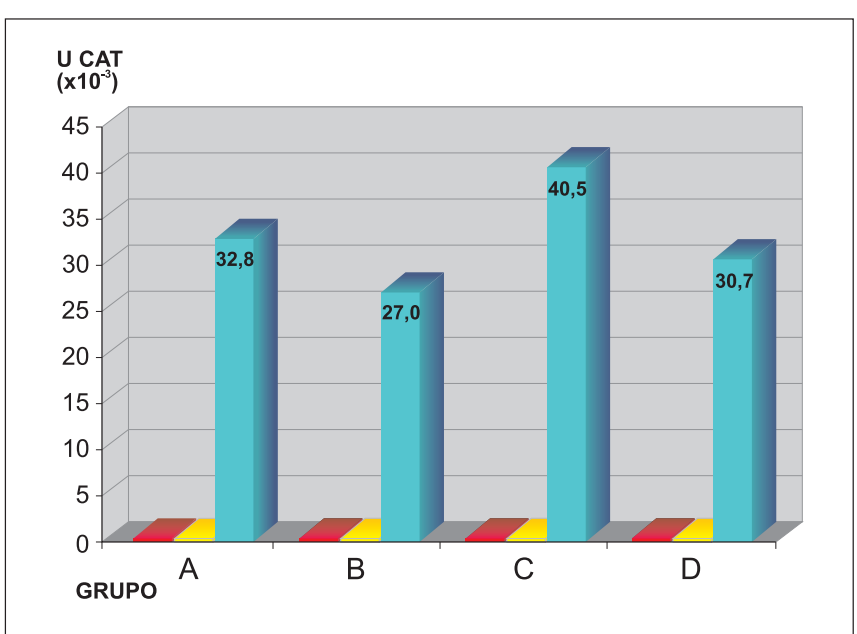

Figura 3. Catalasa en Hígado

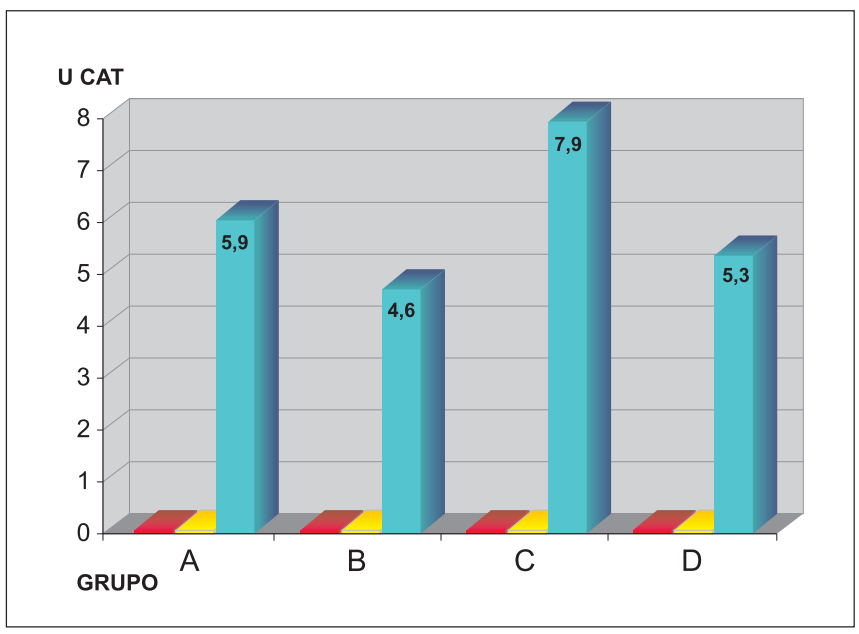

Figura 4. Catalasa en Cerebro

\section{CONCLUSIÓN}

Se concluye que, bajo las condiciones experimentales utilizadas, en el grupo B existe estrés oxidativo como producto del tratamiento dado, manifestándose éste en la disminución de la actividad de SOD y CAT, y que el grupo $C$ luego de haber sido sometido al mismo tratamiento que el grupo B, consiguió una recuperación expresada en el aumento o mantenimiento de los niveles de SOD y CAT, gracias al tratamiento con ejercicio continuo y moderado.

\section{REFERENCIAS BIBLIOGRÁFICAS}

1. Sürmen-Gür E, Erdinc A, Serdar Z, Gür H. Influence of acute exercise on oxidative stress in chronic smokers. JSSM 2003; 2: 98-105.

2. Aslan R, Sekeroglu MR, Tarakçioglu M, Bayiroglu F, Meral I. Effect of acute and regular exercise on antioxidative enzymes, tissue damage markers and membran 
lipid peroxidation of erythrocytes in sedentary students. Tr J Med Sci 1998; 28: 411-414.

3. Del Castillo VC. Antioxidantes, Radicales libres y ejercicio. Educación física y deportes. Julio 2000; 5(23). Disponible en http://www.efdeportes.com/[última revisión Julio 2000].

4. Powers S, Jackson MK. Exercise-Induced Oxidative Stress: Cellular Mechanisms and Impact on Muscle Force Production. Physiol Rev 2008; 88: 1243-76.

5. Gómez-Cabrera MC. Papel de los radicales libres en el ejercicio físico agotador y efecto de la administración de antioxidantes. [Tesis Doctoral]. Departamento de Fisiología. Facultad de Ciencias, Actividad Física y Deportes. Universidad de Valencia, 2004. Disponible en http://www.tdx.cesca.es/TDX-0519105-120702/ [Última revisión Julio 200o].

6. Adriazola MA, Olivera PL. Enzimas Antioxidantes en sujetos de Altura. Tesis para optar al título profesional de Químico Farmacéutico. Facultad de Farmacia y Bioquímica, UNMSM. Lima, 2005.

7. Berrocal N, Valdez A. Niveles Plasmáticos de MDA como indicador de la Peroxidación Lipídica en cobayos de Altura. Tesis para optar al Título Profesional de Químico
Farmacéutico. Facultad de Farmacia y Bioquímica, UNMSM. Lima, 2002.

8. Pajuelo M, Yamada L. Enzimas Antioxidantes en cerebros de cobayos de altura. Tesis para optar al Título Profesional de Químico Farmacéutico. Facultad de Farmacia y Bioquímica, UNMSM. Lima, 2003.

9. Vicente M, Miñano M. Determinación de algunos antioxidantes en sujetos de altura. Tesis para optar al Título Profesional de Químico Farmacéutico. Facultad de Farmacia y Bioquímica, UNMSM. Lima, 2002.

Manuscrito recibido el: 14/12/2009

Aceptado para su publicación el: 16/o8/2010

\section{Correspondencia:}

Nombre: Elena Benavides Rivera

Dirección: Jr. Apurímac 3634 Lima 34 / Jr. Puno 1002 Lima o1 - Perú

Correo electrónico: elenabenavidesr@gmail.com, ebenavidesr@unmsm.edu.pe 